\title{
Use of Applet and Servlet Communication Technique to Administer Online Examinations
}

\author{
Daniel J. Farkas and Narayan Murthy \\ Pace University, Pleasantville, NY, USA

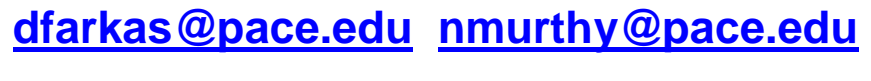

Abstract

Internet and Java technology have developed enormously in recent years. These dramatic developments have provided opportunities and challenges in all fields, particularly in the field of education. What we could not imagine doing just a couple years back has been made possible by these technologies. This short paper illustrates one such example. The example we will discuss is an examination system that a student takes online. When done, the system displays his/her score, and stores the name of the student and his/her score in a file on the server. By clicking another button the students can see answers to all the questions. The way we have done, all the processing is done on the client side and then the result and the name are sent to the server. The server stores the name and score of the student in a file on the server.

A Java applet does the client side computation and a Java Servlet does the processing on the server side. The paper illustrates the power of applet servlet communication.

Keywords: Java Applets, Java Servlets, Ecommerce, CGI Scripts, Perl, HTML, Web, Online assessment

\section{Introduction}

Developers use Java applets to make their Web pages more interactive and functional. Java applets are small Java programs, which are compiled to create platform independent bytecode. The resulting bytecode is (generally) saved on a server. The HTML tag APPLET is used to include a reference to the bytecode file. When the browser downloads the HTML page, the applet bytecode is also downloaded and the Java Virtual Machine (JVM) built-into the browser executes the bytecode resulting in the display of the applet within the browser screen. Thus, Java applets are executed on the client side.

Java servlets, on the other hand, are server side executables. Java servlets are becoming more and more important in industry. Java servlets are the workhorses on ecommerce Web servers that process the incoming orders from the clients. Servlets can do whatever CGI scripts can do, and do better. Java servlets are small programs, which are capable of retrieving the data sent by the client, get connected to a database if necessary and process the data.

Generally, a form built into an HTML document invokes a servlet. Any information sent back by the servlet to the client is generally sent as an HTML document, which is displayed in the usual way by the client browser. As the HTTP is a stateless protocol, the HTML page sent by the servlet is treated a new document and is loaded as a new HTML page.

Material published as part of these proceedings, either on-line or in print, is copyrighted by Informing Science. Permission to make digital or paper copy of part or all of these works for personal or classroom use is granted without fee provided that the copies are not made or distributed for profit or commercial advantage AND that copies 1) bear this notice in full and 2) give the full citation on the first page. It is permissible to abstract these works so long as credit is given. To copy in all other cases or to republish or to post on a server or to redistribute to lists requires specific permission from the publisher at publister@intormingscience.org
Instead of making this transaction HTML based interface, if we make it an applet interface, the displays will be much more elegant and seamless. You really have to experience it to appreciate the elegance of the display of the transaction.

This paper illustrates this feature by implementing an online examination system. Our system admin- 
Use of Applet and Servlet Communication Technique

isters the examination online, grades it and stores the result in a file on the server.

\section{Online Examination System}

In our system, the questions and the key are included in the Java applet.

The starting point for our system is the following page, displayed when the student is ready to take the examination.

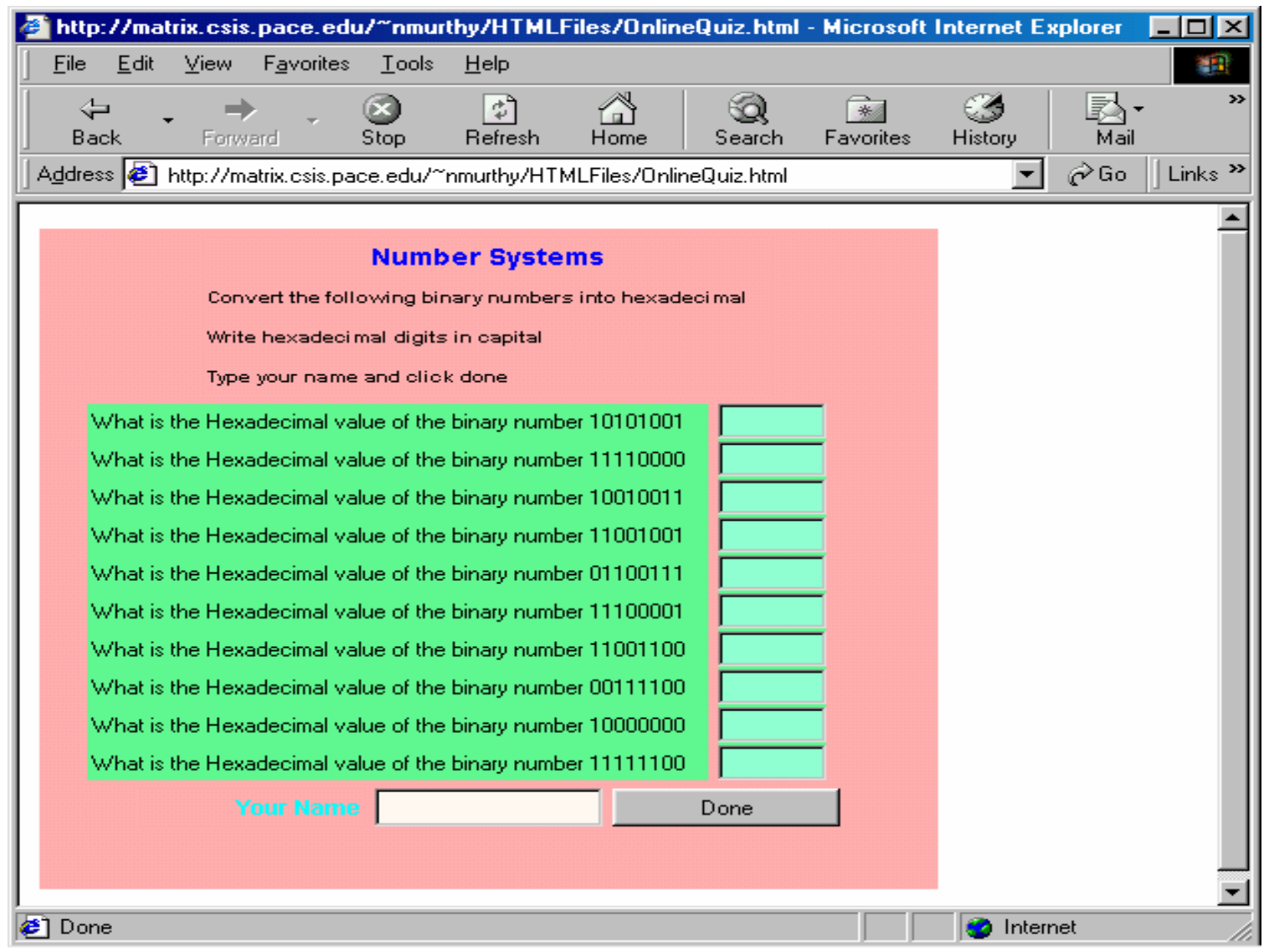

This is an applet. The student types his/her answers to the questions in the text boxes, types his/her name and clicks the Done button.

When the Done button is pressed, the applet does the following:

- Computes how many answers are correct

- Displays the number of correct answers (score)

- Places a * next to incorrect answers

- Sends the name entered and the score to the servlet

- Displays a button "Get Answers" button

The resulting page is shown below: 
Farkas \& Murthy

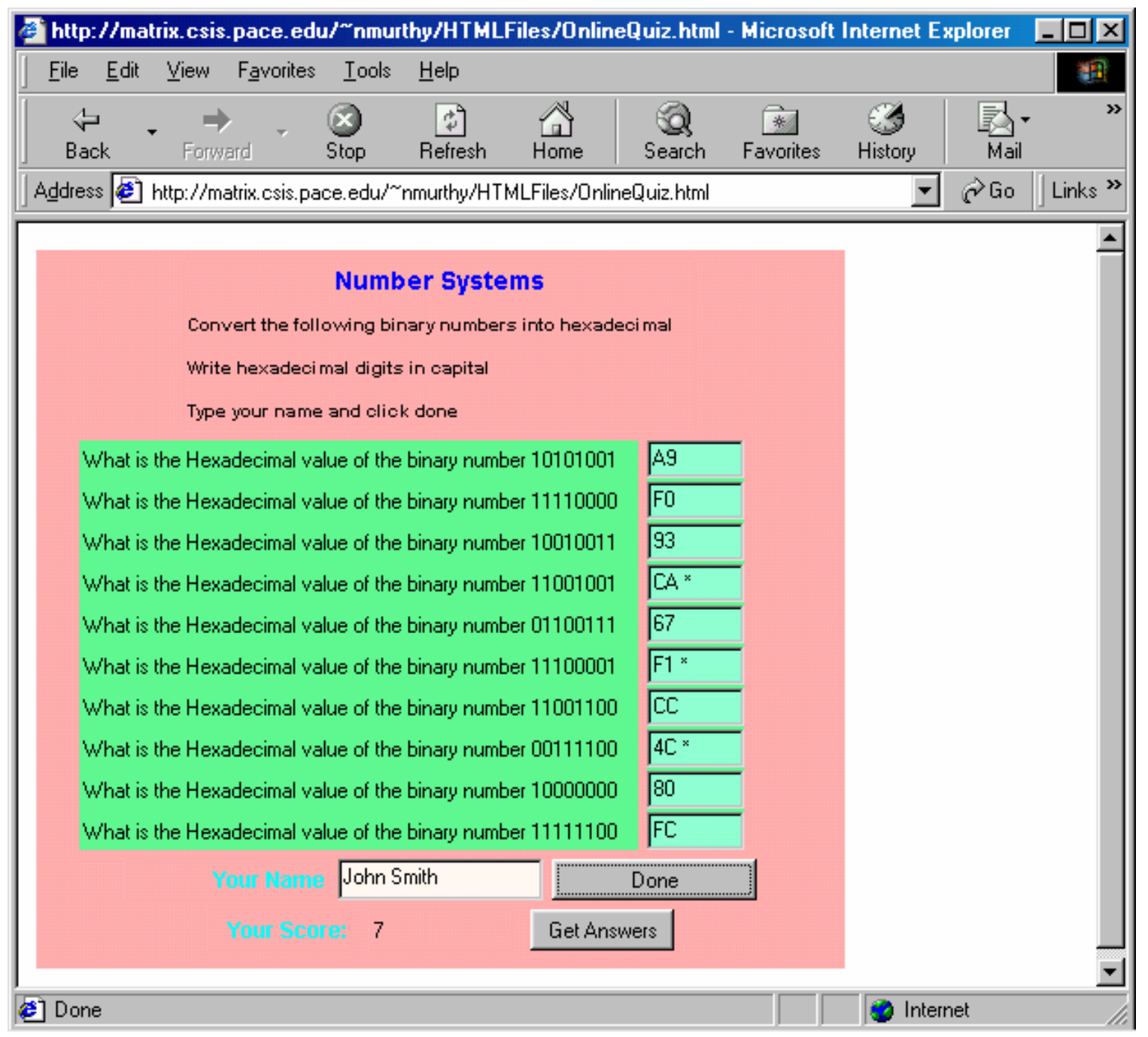

The nice part of this transition is that a page is not loaded afresh, but the same page is modified smoothly to display new information. This smoothness is an integral part of applets. We use hiding/showing components features of Java applets to accomplish this.

The student can click the Get Answers button to see all the correct answers: 
Use of Applet and Servlet Communication Technique

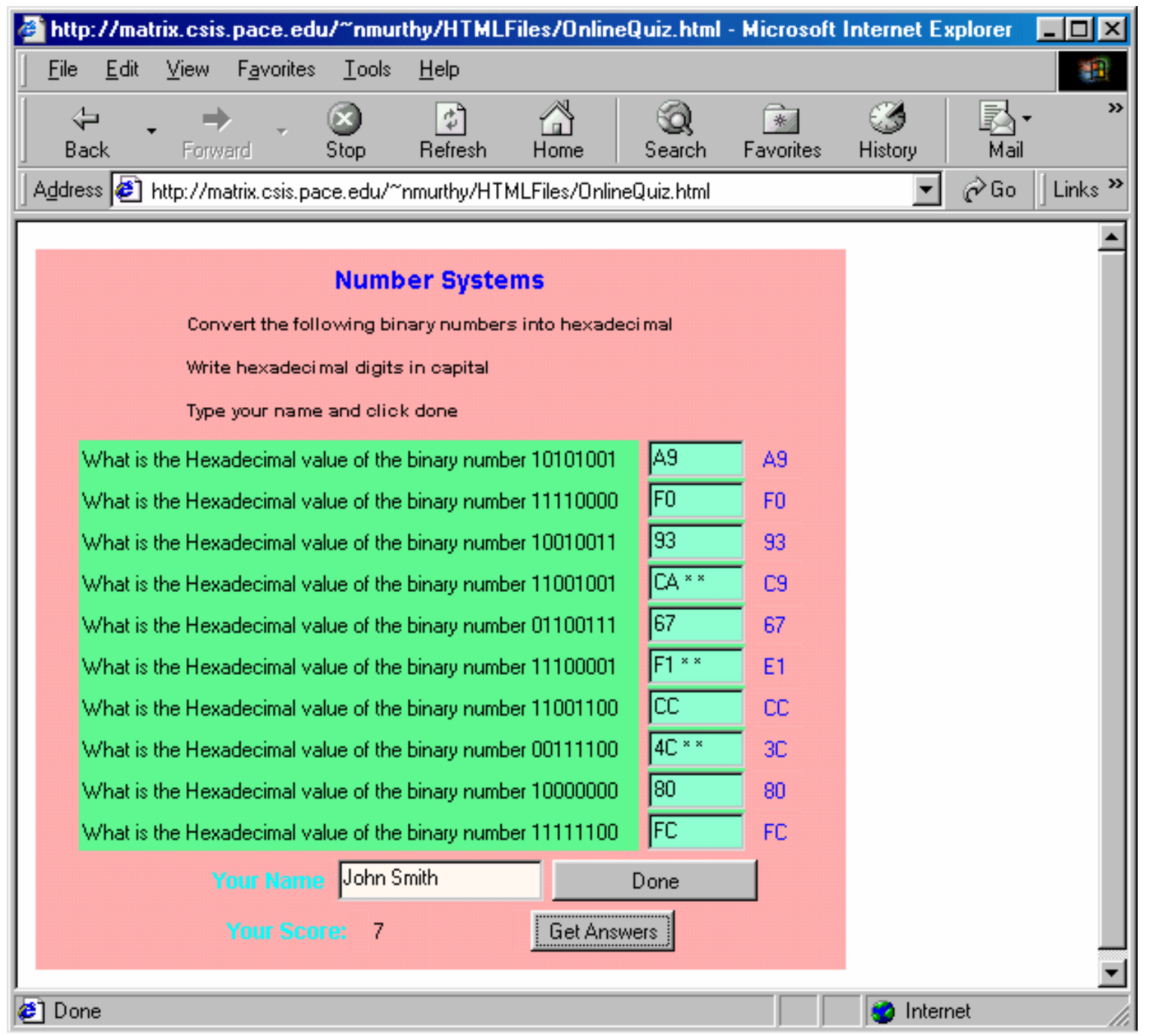

The student, of course can correct his/her mistakes and submit again. But, our servlet records scores from every attempt.

Now, let us turn our attention to the server side. When the user clicks the Done button, the applet invokes the servlet on the server and provides data, namely the name typed in the text box and the score, to the program. The servlet opens a file and saves the information in the file. The servlet also stores the date the data was entered. Here is a look at the file after "John Smith" took the exam: 


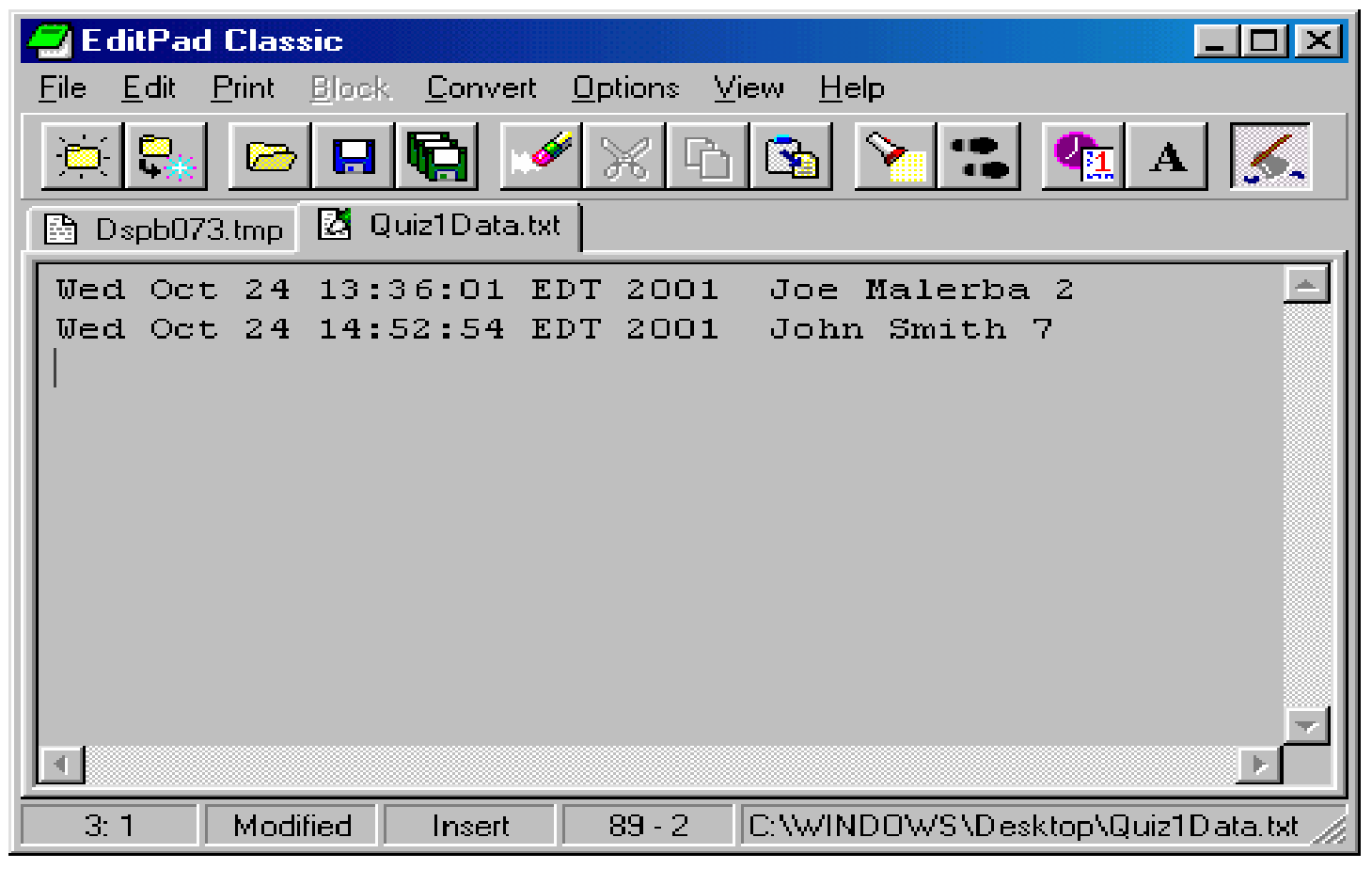

\section{The Applet}

The questions and the key are included in the Java applet program. The series of questions as Label components and the key as an array. Most of what you see on the applet are various Component objects, such as text boxes, labels, Strings, and buttons. We use several layout managers to arrange the component sin a nice way. We handle the event in the usual way by implementing the interface ActionListener, and in particular the actionPerformed() method. The computation of the grade is done in the actionPerformed() method by comparing the answers given by the student to the answers in the key array.

The crucial segment of the applet program is when the applet establishes a connection to the server, invokes the Java servlet on the server and feeds the data to the servlet. The following is that segment:

URL servletUrl= null;

URLConnection connection;

String servletName="http://matrix.csis.pace.edu/nmurthy/servlets/OnlineQuizServlet";

try

\{

servletUrl $=$ new

URL(servletName+"?name="+name+"\&score="+score+"\&ScoresFile="+ScoresFile);

connection $=$ servletUrl.openConnection();

connection.setUseCaches(false);

BufferedReader buf $=$ new BufferedReader(new inputStream-

Reader(connection.getInputStream()));

\}

catch(Exception e)

\{

System.out.println("Exception caught..." + e);

\} 
Use of Applet and Servlet Communication Technique

First a URL object representing the URL for the servlet is created. We have named this object servletURL. This URL object consists the following pieces of information in that order:

$>$ The server domain name

$>$ The directory on the server where the servlet is located

$>$ The name of the servlet

$>\mathrm{A}$ ?

$>$ Name and value pair for each piece of data

In our case,

$>$ The domain name is matrix.csis.pace.edu

$>$ The servlet is located in the directory nmurthy/servlets

$>$ The name of the servlet is OnlineQuizServlet

$>$ Name value pair is name $="+$ name $+" \&$ score $="+$ score $+" \&$ ScoresFile $="+$ ScoresFile

Here, we are sending three pieces of data to the servlet: name, score and file name to save name and score. Values for each of these is sent in the form name=value. The servlet retrieves the data using the name. The delimiter between "name $=$ value" is \&.

Once the servletURL is defined, a connection to the servlet is established using the statement

$$
\text { connection = servletUrl.openConnection }() \text {; }
$$

This invokes the servlet. The servlet sends any data back to the applet as a stream. This data, if any, is processed and formatted by the applet and displayed appropriately. In our example we don't have any user usable data sent by the servlet.

\section{The Servlet}

Our servlet is a very simple one, which extends, the usual, HttpServlet. We extend the doGet(), method. We retrieve the values sent by the applet corresponding to the names "name", "score" and "ScoreFile." The HttpServletRequest method getParameter() is used to retrieve these values:

String name = request.getParameter("name");
int score=Integer.parseInt(request.getParameter("score"));

String ScoresFile=request.getParameter("ScoresFile");

In our example we create a Date object, Date date $=$ new Date ()$;$, to write to the file to indicate when the quiz was taken by the student.

The servlet opens (creates, if it is the first time) a file with the name sent by the applet (ScoreFile) and writes the three items: date, name and score:

String fileName="/home/nmurthy/public_html/servlets/QuizResults/"+ScoresFile;

FileWriter resultsFile=new FileWriter(fileName,true);

PrintWriter toFile=new PrintWriter(resultsFile,true);

toFile.println(date+" "+name+" "+score);

toFile.close(); 


\section{Conclusion}

The new technology has provided faculty very powerful and easy to use tools. The concept discussed in this short paper can be used in many different ways to make faculty more productive. Examples include conducting a survey, evaluation of course at the end of the semester and so on.

\section{References}

Cowell, J. (1999). Essential Java 2 Fast : How to Develop Applications and Applets With Java 2. Springer Verlag.Hall, M. (2000). Core Servlets and JavaServer Pages (JSP). Prentice Hall.

Hunter, J. \& Crawford, W. (2001). Java Servlet Programming. O’reilly.

\section{Biographies}

Daniel Farkas has a MS in Computer Science from NYU, and is currently the Chairman of the Information Systems Department at Pace University in New York. He has been teaching for over 25 years both graduate and undergraduate Information Systems and Computer Science courses. Prior to coming to Pace he spent several years designing computer-based financial systems for the banking industry. In addition to publication in academic proceedings, he is the author of two books, data Communications: Concepts, Terms, Definitions, Carnegie Press, 1982 and Unix for Programmers, John Wiley \& Sons, 1988.

Narayan Murthy has a Ph.D. in Mathematics and a MS in Computer Science from University of Rhode Island, RI, USA. He has taught mathematics and computer science for more than 25 years, in India and USA. He is currently professor and chairman of the department of computer science at Pace University in New York. 